\title{
Optimal configuration of energy storage system in multi-station fusion mode
}

\author{
Zhendong Du ${ }^{1}$, Guosheng Jin ${ }^{1}$, Yu Fang ${ }^{1}$, Chenyin $\mathrm{Yu}^{1}$, Yupeng $\mathrm{Hu}^{1}$, Chuangxin Guo ${ }^{2 *}$, Chutong Wang ${ }^{2}$ \\ ${ }^{1}$ Zhejiang Huayun Electric Power Engineering Design \& Consultation Co, LTD, Hangzhou, Zhejiang, 310014, China \\ ${ }^{2}$ Zhejiang University, Hangzhou, Zhejiang, 310063, China
}

\begin{abstract}
Multi-station fusion mode (MSF) generally includes energy storage system, data center and electric vehicle charging station. It can improve the utilization rate of land and power distribution resources of urban substations. This paper studies configuration of ESS in the MSF model, aiming at reducing total cost. Firstly, an AC-DC system of MSF model is established. Then, aiming at economy, the optimal configuration model of ESS is established. Finally, the effectiveness of the model is verified by a practical example, and the MATLAB toolbox YALMIP with the CPLEX solver is used to conduct the ESS planning.
\end{abstract}

\section{Introduction}

In recent years, with the accelerated transformation of energy to clean and low-carbon and the rapid development of information industry, electric vehicles, 5G communication base stations, data center, distributed power generation and energy storage system (ESS) are booming in urban area [1]. The demand for land resources, power transformation and distribution resources is growing rapidly. The power supply resources and spare space resources of urban substations can be used to build electric vehicle charging stations and data centers to realize intensive development of resources. The construction of multi-station fusion (MSF) can improve the utilization rate of land and power distribution resources of urban substations. Meanwhile, the resource endowment of each station can be combined to realize functional integration [2].

MSF model generally includes ESS, data center and electric vehicle charging station. ESS has multiple values in the multi-station fusion system. For the power grid, the energy storage power station mainly provides auxiliary service functions such as emergency support, peak and frequency regulation and renewable energy consumption [3]. For the data center, the main function of the energy storage power station is to reduce the operation electricity cost [4]. That is, the peak-valley electricity price policy is adopted to transfer the electricity consumption during the peak period to the trough period of the data center to reduce the electricity cost of the data center. For electric car charging stations to come. The main function of the ESS is to shift the peak and fill the valley, and reduce the charging cost by using the difference between the peak and valley electricity prices. At the same time, the ESS also has the function of reducing the peak load of charging, reducing the distribution capacity and improving the utilization rate of equipment.

Few paper study this new model. In [4], the fusion station operation architecture covering data center and ESS was designed. In [5], a multi-station integrated power supply system for assisting peak regulation and autonomous operation is proposed to meet the demand of power grid peak regulation and reserve capacity with large-scale interconnection of distributed generation.

This paper studies configuration of ESS in the MSF model, aiming at reducing total cost. Firstly, a AC-DC of MSF model is established. Then, aiming at economy, the optimal configuration model of ESS is established. Finally, the effectiveness of the model is verified by a practical example, and the MATLAB toolbox YALMIP with the CPLEX solver is used to conduct the ESS planning.

\section{MSF AC/DC system}

In the MSF mode, the ESS has economic value when it plays the functions of peak-shifting, valley filling and consuming renewable energy. The ESS has high equipment utilization rate and good economic benefit when moving peak and filling valley. It is worth noting that ESS are of great value in replacing UPS battery systems in data center. In order to ensure the reliability of power supply, the data center is equipped with a considerable scale UPS battery system, which is used for backup power supply in case of mains power failure. Its utilization rate is very low, and it becomes a "sleeping asset". ESS is used instead of UPS, part of the capacity of the battery is used for peak-shifting and valley filling, and the remaining capacity is used as the backup power supply. The rich capacity of the ESS is used as the backup power supply, which gives full play to the comprehensive benefits of the battery, saves a lot of investment, and has good economic benefits. 
The function of the energy storage station should be positioned to shift the peak and fill the valley for the data center and charging station, while taking into account the replacement of UPS. According to the above functional positioning, the typical system design of an ESS is shown in Figure 1. The energy ESS is located in the data center and replaced by the original UPS. During normal operation, the alternating current is changed into direct current after the filter, which is divided into two ways. After passing through the inverter one way, IT supplies power to IT equipment. The other road enters the energy storage power station to supplement the loss caused by the spontaneous electricity of the ESS. When the mains power fails, the ESS supplies power to IT equipment after passing through the inverter. PCS is set between the 400 $\mathrm{V}$ bus and the energy storage power station to play the function of peak-shifting and valley filling of the energy storage power station. During the low power consumption period, the bus passes through the PCS direction.

The energy storage power station shall guarantee the data center's standby capacity for 30 minutes at any time. The remaining capacity shall be used for peak-shifting to the charging station and the data center to fill the valley.

From the perspective of economy priority, this scheme integrates the AC and DC power supply of the whole station to save investment and operation costs, as well as ensuring the reliability of power supply.

In this scheme, UPS of data center is combined with ESS. ESS were installed in 380v AC bus and 750v DC bus. $\mathrm{DC}$ is supplied by the double road power, power still all the way through the isolation transformer and $\mathrm{AC} / \mathrm{DC}$ conversion from $380 \mathrm{v}$ AC system. The other power supply is from the $750 \mathrm{v}$ DC bus by DC/DC conversion, to replace the original UPS power supply. The large-capacity ESS can also carry out peak-valley arbitrage with high storage and low production, and participate in auxiliary services to obtain profits. The AC-DC system scheme is shown in Figure 2.

Compared with the scheme I, the ESS of the DC bus is used as the second power supply of the data center. When the power grid fails, the AC/DC microgrid runs off-grid. At this time, according to the information such as the ESS SOC, the AC system side or the DC bus side can supply power to the data center to maintain the operation of the data center. When the AC bus and AC/DC converter fail, or the $\mathrm{DC}$ bus and $\mathrm{DC} / \mathrm{DC}$ converter fail, another power supply can still maintain the power supply to the data center. Its reliability can be guaranteed to some extent.

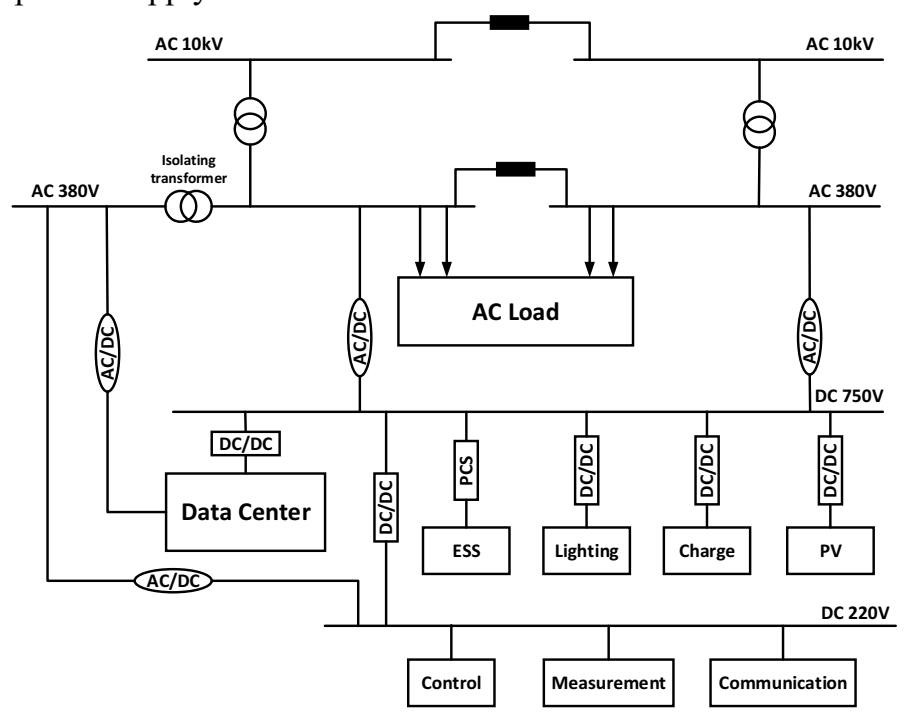

Figure 1. MSF AC/DC system.

\section{Optimal configuration model of energy storage system in MSF}

\subsection{Target function}

The objective of ESS planning is to minimize the overall cost over a full life cycle, including the investment cost of ESS, the operating cost to meet demands and the fixed cost. Considering the time value of the funds and count all costs into equal annual values, the planned target function can be represented as:

$$
\min C=k_{p a} I C+O C+F C
$$

Where $C$ denotes the total cost of annual year; $I C$ denotes the initial investment cost; $O C$ denotes the annual operated cost, $F C$ denotes the annual maintenance cost that is a fixed cost, $k_{p a}$ denotes annual value coefficient. In the annual interest rate is $r$ rate, the life period is the $y$ year, its expression is:

$$
k_{p a}=\frac{r(1+r)^{y}}{(1+r)^{y}-1}
$$

\subsubsection{Cost of investment}

The investment plan for multi-energy storage in the integrated energy network mainly consists of HSS and hydrogen supply chain equipment

$$
\begin{gathered}
I C=k_{1} P^{\max }+k_{2} C_{E S S}^{\max } \\
C_{E S S}^{\max }=C_{\text {back }}+C_{r e}^{\max }
\end{gathered}
$$


$k_{1}, k_{2}$ denote investment coefficient per unit of ESS rated power and rated capacity respectively. $P^{\max }$ denotes ESS rated power. $C_{E S S}^{\max }$ denotes ESS rated capacity. $C_{\text {back }}$ denotes backup capacity of data center.

$C_{r e}^{\max }$ denotes maximum available capacity.

\subsubsection{Operation cost}

MSF model is same as the microgrid. In the MSF, operation cost refers to the cost of purchasing electricity from the main grid, which is expressed as follows:

$$
O C=\sum_{s=1}^{S} \sum_{t=1}^{T} d_{s}\left(\lambda_{t}^{e} \cdot P_{i n}^{e}\right)
$$

Where $S, T$ denote the number of typical days and the number of scheduled hours respectively. $d_{s}$ denotes the total number of days corresponding to the typical day, $\lambda_{t}^{e}$ denotes the purchase price from main grid, $P_{i n}^{e}$ denotes the purchase power.

\subsection{Constraints}

\subsubsection{Power balance:}

$$
P_{i n}^{e}(t)+P_{P V}(t)+P_{E S S}(t)=P_{\text {eload }}(t)+P_{d}(t)
$$

Where $P_{i n}^{e}(t)$ denotes the power purchased form main grid at time t. $P_{P V}(t)$ denotes the output power of $\mathrm{PV}$ at time t. $P_{E S S}(t)$ denotes the power of ESS. Charging is negative, and discharging is positive.

\subsubsection{Data center constraints}

The electricity load of data center is mainly the electricity load of IT equipment, which is generally relatively stable, and the electric energy used is basically converted into heat energy. Refrigeration unit is responsible for transferring this part of the heat energy to the outdoor. Its energy efficiency ratio changes with the outdoor temperature. Generally, the energy efficiency ratio of refrigeration unit system decreases by $1.5 \%$ for every $1{ }^{\circ} \mathrm{C}$ increase in the outdoor temperature. The data center power load can be approximated by the following engineering empirical formula.

$$
P_{d}=P_{I T}+\frac{P_{I T}}{C O P \times[1+0.015 \times(35-t)}
$$

Where, $P_{d}$ is the power load of the data center. $P_{I T}$ is the power load of IT equipment. COP is the energy efficiency ratio of refrigeration unit system. $\mathrm{T}$ is the outdoor temperature.

\subsubsection{ESS constraints}

$$
\begin{gathered}
S O C_{t}^{e}=\left(1-\delta_{e}\right) S O C_{t-1}^{e}+\Delta t\left(\eta^{e, C} P_{t}^{e, C}-\frac{P_{s, t}^{e, F}}{\eta^{e, F}}\right) / C_{e}^{\max } \\
S O C_{\min }^{e} \leq S O C_{t}^{e} \leq S O C_{\max }^{e} \\
\delta_{\mathrm{c}}+\delta_{d} \leq 1 \\
0 \leq P_{t}^{e, C} \leq \delta_{\mathrm{c}} \cdot P_{e}^{\max } \\
0 \leq P_{t}^{e, \mathrm{~F}} \leq \delta_{d} \cdot P_{e}^{\max } \\
P_{t}^{e, C}+P_{t}^{e, \mathrm{~F}}=P_{E S S}(t)
\end{gathered}
$$

Where $S O C_{t}^{e}$ denotes the storage state of the ESS at time t. $\eta^{e, C}, \eta^{e, F}$ denote the ESS charge and discharge efficiency respectively. $\delta_{e}$ denotes the self-draining efficiency of the ESS, $S O C_{\min }^{e}, S O C_{\max }^{e}$ denote the upper and lower limits of $S O C_{t}^{e}$ respectively. For ESS. $\delta_{\mathrm{c}}, \delta_{d}$ are $0-1$ variables of the ESS charge state and discharge state respectively. Since the ESS cannot be charged and discharged at the same time, the sum of charge state and discharge state is less than or equal to 1 .

\section{3 solve method}

This article calls the CPLEX solver within Matlab to solve the above optimization model.

Through the necessary means of control, $\mu$ and $\lambda$ can take a fixed value. To clearly describe the algorithm's process, the model is unified in a compact form, resulting in a linear planning model in the following form:

$$
\begin{gathered}
\text { obj. } \min f(\mathbf{x}) \\
\text { s.t. } \mathbf{A}_{\text {eq }} \mathbf{x}=\mathbf{b}_{\text {eq }} \\
\mathbf{A x} \leq \mathbf{b}
\end{gathered}
$$

where $\mathbf{x}$ denotes the control variable and the status variable.

\section{Case study}

\subsection{Study scenes and parameters}

This study design an actual multi-station fusion substation. According to the average temperature in Beijing over the years, the daily curve of a typical data center is plotted, as shown in Figure 2, where IT equipment in the data center. The average load is $350 \mathrm{KW}$, the refrigeration unit system rated COP is 3.5 , the daily average load is $420 \mathrm{KW}$, the maximum load is $426 \mathrm{KW}$, and the minimum load is 416 $\mathrm{KW}$. Other electrical load is shown in Figure 3. The PV output power is shown in Figure 4

The investment factor $\mathrm{k} 1$ is taken to 420 yuan $/ \mathrm{kW}$. $\mathrm{k} 2$ is taken to 1400 yuan $/ \mathrm{kW}$. The initial SOC of ESS is set to 0.2. The upper and lower limits of the SOC are 0.2, 0.9, 
respectively, ensuring that the energy storage device is not overcharged. The self-emission rate of ESS is taken as $10^{-}$ ${ }^{8}$. The fixed cost allocation to the annual cost is set at 10000 yuan.

In the study, the investment planning period for all equipment is 10 years. The one-day schedule time is 24 hours. The annual interest rate is taken to $5 \%$. The time period division and the price of electricity in each period are shown in Table 1 from Jiangsu province.

The parameters of the original equipment in the in the study scenario is set as follows:

case 1: MSF do an optimal configuration of ESS by the method proposed by this paper.

case2: No energy storage is installed in MSF model.

Table 1. Formatting sections, subsections and subsubsections.

\begin{tabular}{lll}
\hline & \multicolumn{2}{l}{ Price of each period $($ Yuan $/(\mathrm{kW} \cdot h))$} \\
\cline { 2 - 2 } peak & $08: 00-12: 00$ & 1.10 \\
& $17: 00-21: 00$ & \\
flat & $12: 00-17: 00$ & 0.66 \\
& $21: 00-24: 00$ & \\
valley & $00: 00-8: 00$ & 0.32 \\
\hline
\end{tabular}

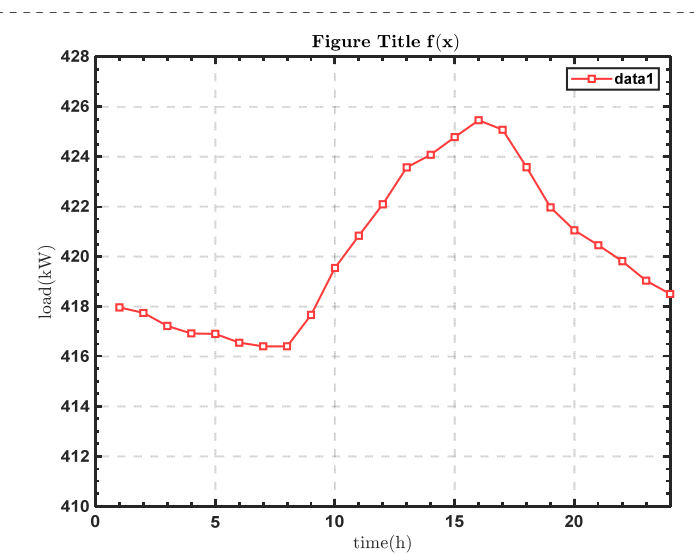

Figure 2. Typical load curve of data center.

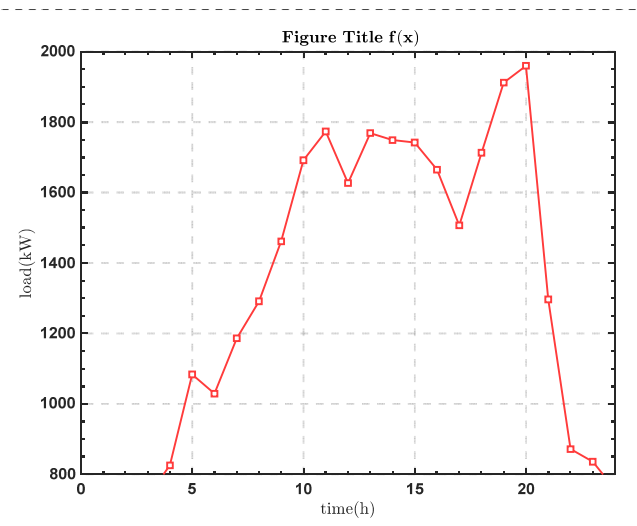

Figure 3. Typical load curve of data center.

\subsection{Study Results}

Table 2. Study results .

\begin{tabular}{llll}
\hline & \multicolumn{1}{c}{ Case1 } & Case2 \\
\cline { 2 - 3 } ESS rated power $/ \mathrm{kW}$ & 2880 & 0 \\
ESS rated capacity/kWh & 4113 & 0 \\
$\begin{array}{l}\text { investment cost /million } \\
\text { yuan }\end{array}$ & 0.78 & 0 \\
$\begin{array}{l}\text { operation cost / million } \\
\text { yuan } \\
\text { fixed cost / million yuan }\end{array}$ & 1 & 182.33 \\
total cost / million yuan & 98.2 & 0 \\
\hline
\end{tabular}

\section{3 conclusion}

The reason of resulting in a lower total cost than another case is the optimal configuration method proposed in this paper. Planning and construction of ESS in MSF model can affect the total cost of the whole system, and using this method to choose a more reasonable configuration can increase the system revenue and reduce the total cost of the system. Further research can be carried out on the participation of ESS in MSF model. 


\section{Acknowledgments}

This work is supported by Technology Project of Zhejiang Huayun Electric Power Engineering Design \& Consultation Co., Ltd.(2019C1D09P01)

\section{References}

1. Kwon, S., Ntaimo, L., Gautam, N. (2019). Demand Response in Data Centers: Integration of Server Provisioning and Power Procurement. IEEE Transactions on Smart Grid 10: 4928-4938.

2. Ma Huimeng, Li Xiangjun, Jia Xuecu. (2021) Capacity Configuration and Coordinated Operation Strategy in the Multi-station Integration Scenario. Electric Power Construction, 42: 96-104.

3. MA Hengrui, WANGBo, GAO Wenzhong, et al. (2018) Operation optimization of regional integrated energy system participating in regulation service based on pay for performance. Automation of Electric Power Systems, 42: 127-135.

4. Meng Chao, Liu Wenliang, Yang Qi, et al.(2020) Research on Operation Optimization of Edge Data Center Under the Background of "Multi-station Integration. Advanced Engineering Sciences, 52: 4955.

5. Yan, C., Wei, J., Wenbin, W., Huibin, L., Zhijie, S. (2020). Multi-station fusion power supply system to assist peak regulation strategy. Power System Protection and Control 48, 57-65. 